\title{
SURFACE FLOW MODIFICATION OF AEROFOILS FOR AUTOMOTIVE RACING CAR APPLICATIONS
}

\author{
Richard Allarton, Jun Yao*, Tyler Clifford, Benjamin Hitchborn, \\ Liam J Parker, Joshua Shaw \\ School of Engineering, University of Lincoln, Brayford Pool, Lincoln LN6 7TS, UK \\ *Correspondence: jyao@lincoln.ac.uk
}

\begin{abstract}
An aerofoil commonly used in aerospace engineering to produce lift is also employed in the motorsport industry to produce downforce for improving traction during cornering. This paper investigates aerofoil surface modification through 'golf ball dimpling', used to reduce flow separation behind a golf ball. The studies of other researchers have shown that this type of design can have a positive effect on improving aerofoil performance. However, no optimization information of dimple sizing is given in literature. Therefore, three types of dimpling sized at 5,10 and $15 \mathrm{~mm}$ are applied to the surface of a NACA 6615 wing at $25 \%$ chord length from the leading edge in this study using Computational Fluid Dynamics (CFD) as an initial design process. Then a physical model, made through 3D printing additive manufacturing (AM), is tested at angles of attack (AoA) ranging from $0^{\circ}$ to $20^{\circ}$ and wind speed up to $30 \mathrm{~m} / \mathrm{s}$ in a subsonic wind tunnel. Experimental and CFD results show that the smallest dimple size provides the most significant increase on lift to drag ratio at high AoA above $10^{\circ}$. This ratio increases further with the wind speed, indicating a high AoA wing favours downforce to improve drag reduction performance.
\end{abstract}

Keywords: dimpling, aerofoil, downforce, drag reduction, motorsport, wind tunnel, CFD.

\section{Introduction}

The airfoil is commonly used in the aviation industry to produce lift. However, the automotive and motorsport industries also use airfoil to produce force that pushes the car down (downforce), allowing better traction when the car is turning into a corner [1]. Without using aerodynamic downforce, the way to increase the traction between the wheels and the road can be through increasing the weight of car, but this causes adverse effects, such as reducing the acceleration and maximum speed. Nevertheless, increasing the downforce will increase drag, which will ultimately affect performance, particularly relevant to motorsports. Therefore, a golf ball type of design with dimples is introduced to reduce the drag experienced for a racing car. This is accomplished by creating a turbulence boundary layer around the surface of a golf ball, allowing air flow to move faster to penetrate into the turbulence field and reduce the size of the turbulent wake region left behind of airfoil wing [2]. In order to increase lift, an asymmetric aerofoil wing is chosen to be implemented for racing cars [3]. The viscous effects of air flow over aerofoil are confined to a thin boundary layer around wing surfaces where flow passes around streamlined wing body and dimples, leading to possible flow separation at high angle of attack (AoA). Moreover, the flow motion of the viscous boundary layer becomes slow, as there is insufficient momentum to move fluids into a fast moving flow region, creating a flow recirculation region. While increasing Reynolds number, the viscous boundary layer will enhance turbulence motions further downstream [4]. Therefore, the 
success of current study is heavily reliant on identifying this viscous boundary layer to understand how it can affect flow separation along with the drag.

The theory of dimpling impact on an aerofoil's dynamic performance is far less well understood. In this study, the investigation will focus on the effect of dimpling size on flow behaviour around the wing surface, using 3D additive manufactured models for experimental test in a wind tunnel. The experimental data will be validated by Computational Fluid Dynamics (CFD) predictions.

\section{Design Methodology}

\subsection{Design selection and wind tunnel test}

A NACA 6615 aerofoil was chosen for the study due to high lift coefficient and similarity to that used in motorsports. The chord and span of the aerofoil wing are 260 $\mathrm{mm}$ and $260 \mathrm{~mm}$. Four physical models are used, including a clean wing and wings with $5 \mathrm{~mm}, 10 \mathrm{~mm}$ and $15 \mathrm{~mm}$ dimples (see in Fig.1). The dimples are arranged in three rows to stimulate turbulence. The edge of dimples is chamfered to reduce shear stress around each singular dimple. The material is ABS-M30, owing to its strong, high tensile impact and flexural strength [5]. Experiments were conducted in a subsonic wind tunnel (AF1300 from TecQuipment) to investigate lift and drag forces. The wings were secured with varying inflow AoA at air velocity of $30 \mathrm{~m} / \mathrm{s}$ at $0^{\circ}, 5^{\circ}, 10^{\circ}, 15^{\circ}$ and $20^{\circ}$, respectively.

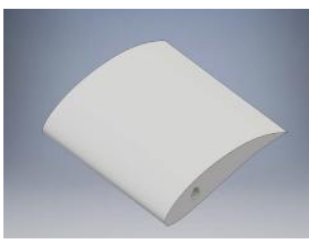

(a) Clean wing-no dimple

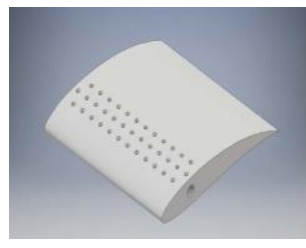

(b) $5 \mathrm{~mm}$ dimples

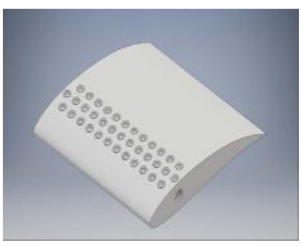

(c) $10 \mathrm{~mm}$ dimples

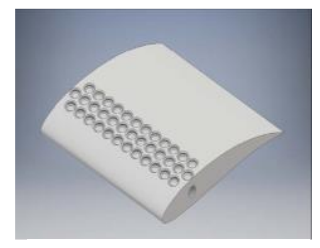

(d) $15 \mathrm{~mm}$ dimples

Figure 1: NACA 6615 with dimples produced using Computer Aided Design (CAD) and 3D printing additive manufacturing (AM) methods.

\subsection{Computational Fluid Dynamics}

The numerical study is carried out using CFD approach to predict the flow feature for aerofoil dimples design. A computational domain of $300 \mathrm{~mm} \times 300 \mathrm{~mm} \times 600 \mathrm{~mm}$ is created using ANSYS software, which has the same size of wind tunnel test section. After mesh independent studies, a fine mesh of 2.1 million tetrahedron cells was employed for CFD model validation. Figure 2 shows an example of 2D plane of computational domains comprising of $0^{\circ}$ and $20^{\circ}$ AoA with $15 \mathrm{~mm}$ dimpled aerofoil wings. 


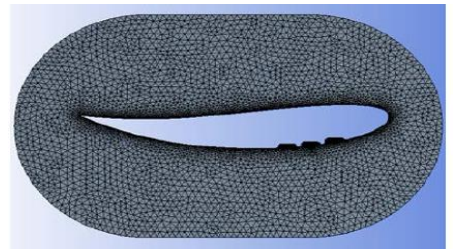

(a) $\alpha=0^{\circ}$

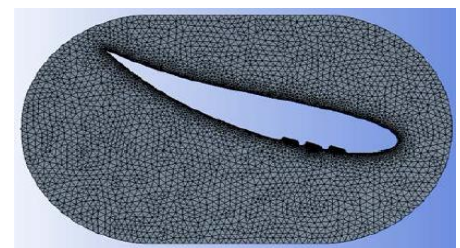

(b) $\alpha=20^{\circ}$

Figure 2: The mesh of $15 \mathrm{~mm}$ dimpled wing. (a) $\mathrm{AOA}=0^{\circ}$; (b) $\mathrm{AOA}=20^{\circ}$.

\section{Results and Discussion}

\subsection{CFD simulation results for NACA 6615 Aerofoil}

The separation of the air flow over the surface of the aerofoil is identified by analysing 2D CFD simulation results. Four different AoAs at $0^{\circ}, 5^{\circ}, 10^{\circ}$ and $20^{\circ}$ were studied using a 2D plane of aerofoil wing with dimples at inflow velocity of $30 \mathrm{~m} / \mathrm{s}$ (see Fig.3). With the increase of wing AoA up to $20^{\circ}$, a clear air flow swirling feature is captured at the upper camber after dimpling point and it extends further into the trailing edge region, where the flow separation can be seen clearly due to vortex shedding, and its intensity increases with the increase of aerofoil AoA up to $20^{\circ}$. The velocity is generally greater on the upper camber and the maximum velocity is observed at the region close to the leading edge, and this is where the dimples are placed to increase the lift to drag ratio. CFD simulations show that dimples placed on the aerofoil surface can trigger earlier flow separation on upper wing surface which starts at $\mathrm{AoA}=10^{\circ}$, compared to the flow performance of a clean aerofoil at same angle attack at which no clear flow separation is predicted. The flow separation point is seen to have a delay and a lateral delay point migrates towards the trailing edge close to wake region [6]

Figure 4a displays a comparison of lift to drag ratio difference between experiment and CFD data at AoA of $0^{\circ}, 5^{\circ}, 10^{\circ}$ and $20^{\circ}$ for aerofoil with a dimple size of $15 \mathrm{~mm}$. A similar trend is seen by both experimental data and CFD with increasing the aerofoil angle of attack. A consistent data is determined at $A o A=10^{\circ}$. The differential value of lift to drag ratio, which has a negative effect, is larger by experiment than CFD prediction at low AoA, and contrary to that observed at high AoA. A decrease of lift to drag ratio is also captured for aerofoil with AoA larger than $6^{\circ}$ (see Fig.4b) in wind tunnel tests. By increasing aerofoil AoA further, no significant impact on lift to drag ratio is observed. The curve fitting has raised a correlation with formula determined at $\mathrm{y}=$ $(49 / 800) \mathrm{x}^{\wedge} 2+(203 / 80) \mathrm{X}-25$

\section{Conclusion}

The trend of CFD prediction and wind tunnel test data is almost identical, i.e. the lift to drag ratio increases with the increase of aerofoil AoA. This implies that aerofoil wing dimples can have a positive impact on improving the lift to drag ratio at high AoA which is beneficial for racing cars to maximize the downforce (inverted lift) and improve the 
cornering speed at the same traction with reduced drag. However, dimpling effect may have limited effect for racing cars having a low AoA wing set up at an optimal top speed.

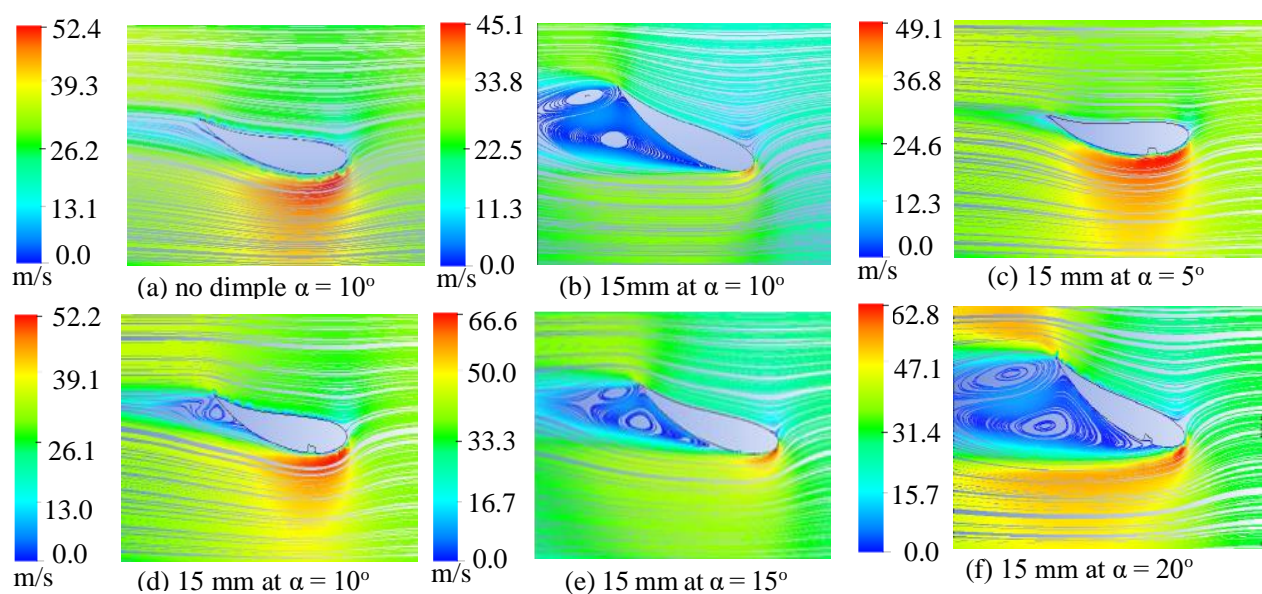

Figure 3: the velocity streamlines superimposed with vector plot to visualise vertex shedding presented by CFD simulation. The inlet velocity is taken $30 \mathrm{~m} / \mathrm{s}$.
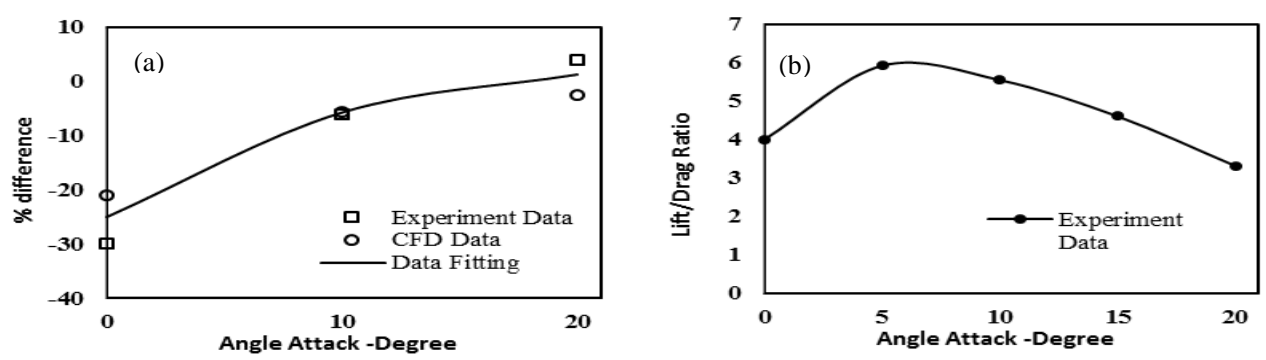

Figure 4: (a). Comparison of difference in lift to drag ratio from wind tunnel test and CFD simulation, the curve fitting formula: $y=(-49 / 800) x^{\wedge} 2+(203 / 80) x-25$; (b), Lift/Drag ratio variation against angle of attack (AoA).

\section{References}

1. Krzysztof Kurec, Michal Remer, Tobiasz Mayer, Sylwester Tudruj, Janusz Piechna. Flow control for a car mounted rear wing. International J. of Mechanical Sciences, Vol.152, 384-399 (2019).

2. S. Mahamuni. A review on study of aerodynamic characteristics of dimple effect on wing. International J. of Aerospace and Mechanical Engineering. 2(4), 18-21 (July, 2015). 3. J. Katz, Aerodynamics of Race Cars, San Diego: University of San Diego (2006). 4. E.L. Houghton, P.W. Carpenter, S.H. Co;;icott, D.T. Valentine. Viscous Flow and Boundary, Aerodynamics for Engineering Students (7th Edition), 151-254 (2017). 5. Dario Croccolo, Massimiliano De Agostinis, Giorgio Olmi. Experimental characterization and analytical modelling of the mechanical behaviour of fused deposition processed parts made of ABS-M30. Computational Material Science, Vol.79, 506-518 (November, 2013). 6. Mohamed Gad-ontrol, Revel-Hak and Dennis M. Bushnell. Separation control: review. J. Fluid Eng. 113(1), 5-30 (May 01, 1991). 